\title{
The pncA gene from naturally pyrazinamide- resistant Mycobacterium avium encodes pyrazinamidase and confers pyrazinamide susceptibility to resistant $M$. tuberculosis complex organisms
}

\author{
Zhonghe Sun, Angelo Scorpio and Ying Zhang
}

Author for correspondence: Ying Zhang. Tel: +1 410614 2975. Fax: +1 4109550105. e-mail: yzhang@phnet.sph.jhu.edu

Department of Molecular Microbiology and Immunology, School of Hygiene and Public Health, Johns Hopkins University, Baltimore, MD 21205, USA
The antituberculosis drug pyrazinamide (PZA) needs to be converted into pyrazinoic acid (POA) by the bacterial pyrazinamidase (PZase) in order to show bactericidal activity against Mycobacterium tuberculosis. $M$. avium is naturally resistant to PZA. To investigate whether this natural resistance to PZA is due to inability of the $M$. avium PZase to convert PZA to bactericidal POA, the $M$. avium PZase gene (pncA) was cloned by using the $M$. tuberculosis pncA gene as a probe. Sequence analysis showed that the $M$. avium pncA gene is 561 bp long, encoding a protein with a predicted size of about $19.8 \mathrm{kDa}$; but Western blotting showed that the $M$. avium PZase migrated as a $24 \mathrm{kDa}$ band when expressed in $M$. bovis BCG and Escherichia coli. Sequence comparison revealed that $M$. avium PZase has $67.7 \%$ and $32.8 \%$ amino acid identity with the corresponding enzymes from $M$. tuberculosis and $E$. coli, respectively. Southern blot analysis with the $M$. avium pncA gene as a probe showed that $M$. terrae, $M$. gastri, $M$. marinum, $M$. fortuitum, $M$. xenopi, $M$. gordonae, $M$. szulgai, $M$. celatum and $M$. kansasii have close pncA homologues, whereas $M$. chelonae and $M$. smegmatis did not give significant hybridization signals. Transformation with the $M$. avium pncA gene conferred PZA susceptibility to PZA-resistant $M$. tuberculosis complex organisms, indicating that the nonsusceptibility of $M$. avium to PZA is not due to an ineffective PZase enzyme, but appears to be related to other factors such as transport of POA.

Keywords: pyrazinamide susceptibility, pyrazinamidase, pncA, Mycobacterium avium

\section{INTRODUCTION}

Mycobacterium avium, a slow-growing saprophytic mycobacterium, is an important opportunistic pathogen in immunocompromised conditions such as HIV infection (Ellner et al., 1991). Like many other mycobacteria, $M$. avium is naturally resistant to many antibiotics. The nonsusceptibility of mycobacteria to antibiotics has been suggested to be due to the impermeability of the mycobacterial cell wall (Jarlier \& Nikaido, 1994). While

Abbreviations: POA, pyrazinoic acid; PZA, pyrazinamide; PZase, pyrazinamidase.

The GenBank accession number for the $M$. avium pncA gene sequence determined in this work is $U 80820$.
M. avium has a similar lipid-rich cell wall structure to Mycobacterium tuberculosis (Jarlier \& Nikaido, 1994), it is nevertheless resistant to some drugs that are active against $M$. tuberculosis, such as isoniazid and pyrazinamide (PZA). In a study by Heifets et al. (1986), $M$. avium was found to be resistant to at least $100 \mu \mathrm{g}$ PZA $\mathrm{ml}^{-1}$. In contrast, $M$. tuberculosis is uniquely susceptible to PZA, with a minimum inhibitory concentration (MIC) of $50-100 \mu \mathrm{g} \mathrm{ml}^{-1}$ (McDermott \& Tompsett, 1954); other mycobacteria or bacterial species are usually completely insensitive to PZA.

In M. tuberculosis, the susceptibility to PZA is correlated with the activity of a single amidase enzyme with both pyrazinamidase (PZase) and nicotinamidase activities (Konno et al., 1967). PZase in M. tuberculosis 
converts PZA to bactericidal pyrazinoic acid (POA) inside the bacterial cell (Bonicke \& Lisboa, 1959; Scorpio \& Zhang, 1996), and it is conceivable that loss of PZase activity could cause PZA resistance in this organism. Many studies have documented the correlation between defective PZase activity and PZA resistance in clinical isolates of $M$. tuberculosis (McClatchy et al., 1981; Trivedi \& Desai, 1987; Miller et al., 1995). We have recently cloned the M. tuberculosis gene $(p n c A)$ encoding an enzyme with both PZase and nicotinamidase activity (Scorpio \& Zhang, 1996) and have confirmed, by transforming resistant bacilli with a functional $M$. tuberculosis $p n c A$ gene, that defective PZase activity resulting from $p n c A$ mutations is the cause of PZA resistance in M. tuberculosis complex organisms (Scorpio \& Zhang, 1996). Furthermore, we have shown that mutation in $p n c A$ is the major mechanism of PZA resistance in M. tuberculosis (Scorpio et al., 1997b; Sreevatsan et al., 1997). Interestingly, $M$. bovis and $M$. bovis BCG - a member of the $M$. tuberculosis complex - were found to have a single point mutation of $\mathrm{C}$ to $\mathrm{G}$ at nucleotide position 169 of the $p n c A$ gene compared with the M. tuberculosis $p n c A$ sequence, causing a substitution of histidine to aspartic acid at amino acid position 57 of the PncA sequence (Scorpio \& Zhang, 1996; Scorpio et al., 1997a). This single point mutation in the $M$. bovis $p n c A$ gene is the cause of the defective PZase activity and natural resistance to PZA in M. bovis and BCG, and has been recently shown to be useful for rapid differentiation of M. bovis from M. tuberculosis (Scorpio et al., 1997a).

However, the correlation between presence of PZase activity and susceptibility to PZA does not hold for other mycobacterial species. For example, unlike $M$. tuberculosis, M. avium has PZase activity (Tarnok \& Rohrscheidt, 1976) but it is not susceptible to PZA (Heifets ei al., 1986). It was not clear whether the natural resistance of $M$. avium to PZA is due to insufficient ability of the $M$. avium PZase to potentiate the PZA activity, or to other potential differences between these organisms in properties such as cell wall permeability or the transport of PZA. To distinguish between the above possibilities, we cloned the $M$. avium $p n c A$ homologue by using the $M$. tuberculosis $p n c A$ gene as a probe and transformed the $M$. avium $p n c A$ gene into PZA-resistant strains of $M$. tuberculosis complex organisms. We found that the $M$. avium pncA gene conferred a high degree of PZA susceptibility upon PZA-resistant $M$. tuberculosis strains. Our result suggests that the nonsusceptibility of $M$. avium to PZA is not due to its having an inefficient PZase enzyme in terms of potentiating PZA activity, but rather to other factors such as the relative impermeability of the $M$. avium cell wall or the transport of PZA/POA.

\section{METHODS}

Mycobacterial strains and DNA. Mycobacteria were grown in 7H9 liquid medium with $\mathrm{ADC}$ (albumin-dextrose-catalase) enrichment (Difco) at $37^{\circ} \mathrm{C}$ for 3-5 $\mathrm{d}$ for fast growers and 3-4 weeks for slow growers. The mycobacterial species $M$. terrae, $M$. kansasii, M. gastri, M. marinum, M. fortuitum, $M$. chelonae, M. xenopi, M. gordonae, M. szulgai and M. celatum were kindly provided by Dr Salman Siddiqi, Becton Dickinson Diagnostic Instrument Systems Inc. Genomic DNA from various mycobacterial species was isolated using glass beads as described previously (Zhang et al., 1992).

PZase enzyme assay and PZA susceptibility testing. PZase activity was assayed according to Wayne (1974). Briefly, a heavy bacterial inoculum was overlaid on the surface of Dubos agar (Difco) in a test tube containing $100 \mu \mathrm{g} \mathrm{PZA} \mathrm{ml}^{-1}$. The inoculated tubes were incubated at $37^{\circ} \mathrm{C}$ for $3-5 \mathrm{~d}$, then $2 \mathrm{ml} 1 \%$ ferrous ammonium sulfate was added to the tubes. After about $2 \mathrm{~h}$ incubation at $4{ }^{\circ} \mathrm{C}$, the results were recorded. Positive PZase activity was indicated if a brownish band appeared on the agar surface. A positive culture (PZA-sensitive M. tuberculosis strain $\mathrm{H} 37 \mathrm{Rv}$ ) and a negative culture (BCG Pasteur, defective in PZase) were included as controls. The susceptibilities to PZA of different bacterial species or strains were determined on $7 \mathrm{H} 11$ agar (Difco) plates in the case of mycobacteria, and on LB agar plates in the case of Escherichia coli. The agar media, which were adjusted to $\mathrm{pH} 5 \cdot 5$, contained $50,100,250,500,1000$ or $2000 \mu \mathrm{g} \mathrm{PZA} \mathrm{ml}^{-1}$. Two dilutions (about $10^{4}-10^{6}$ bacterial cells) of stationary-phase bacterial cultures were spread onto the agar plates, which were then incubated at $37^{\circ} \mathrm{C}$ for $1 \mathrm{~d}$ for E. coli, 3-5 d for fastgrowing mycobacteria, or 3-4 weeks for slow-growing mycobacteria. The minimum inhibitory concentration (MIC) was determined using the absolute concentration method based on no bacterial growth on plates with the lowest concentration of PZA.

Cloning of the $M$. avium pncA gene. The $M$. avium pncA gene was cloned by screening an $M$. avium $\lambda$ ZAPII phage library (NIH AIDS Reagent Program) as described by Sambrook et al. (1989) with a ${ }^{32} \mathrm{P}$-labelled $329 \mathrm{bp}$ PCR product containing part of the M. tuberculosis pncA gene (Scorpio \& Zhang, 1996) as a probe. The $329 \mathrm{bp}$ M. tuberculosis pncA probe was obtained by PCR amplification (Saiki et al, 1988) using the following forward and reverse primers. The forward primer (5'ATCAGCGACTACCTGGCCG3') was taken from 91-109 bp and the reverse primer ( $5^{\prime}$ GCGCACACAATGATCGGTGG3') from $420-400 \mathrm{bp}$ of the $M$. tuberculosis pncA coding sequence (GenBank accession number U59967; Scorpio \& Zhang, 1996). A positive phage clone containing the $M$. avium pncA gene was isolated, amplified, and the phage DNA was extracted as described by Sambrook et al. (1989). To locate the $M$. avium pncA gene, the phage DNA was subjected to restriction enzyme mapping followed by Southern blot analysis with the $329 \mathrm{bp} \mathrm{M}$. tuberculosis pncA PCR product as a probe. A $1.6 \mathrm{~kb} B a m \mathrm{HI}$ hybridizing fragment from the pncAcontaining phage DNA was subcloned into pUC19. Standard molecular cloning techniques were carried out as described by Sambrook et al. (1989).

DNA sequence analysis. The complete $M$. avium $p n c A$ gene sequence was determined for both strands from the $1.6 \mathrm{~kb}$ BamHI-pUC19 construct by primer walking in an automatic DNA sequencer (ABI model 377) at Johns Hopkins Genetic Core Facility. The multiple sequence homology alignment among $M$. avium, $M$. tuberculosis and $E$. coli PncA sequences was performed using the CLUSTAL method.

Southern blotting analysis. Southern blotting analysis of mycobacterial genomic DNA was performed as described previously (Zhang et al., 1992). Briefly, genomic DNA from various mycobacterial species was isolated and digested with 
Bam HI, and run on $0.8 \%$ DNA agarose gel. Genomic DNA fragments from the gel were transferred onto nylon membrane by vacuum blotting, and the membrane was fixed by UV. The DNA probe for the Southern blotting analysis was prepared by a PCR approach using primers derived from within the $M$. avium pncA gene. The forward primer (5'GCATCAACGCCTACCTGGAC3') was taken from 87-107 bp, and the reverse primer ( $5^{\prime}$ TGCACCAGCACCCGGGTGGT3') from 474-455 bp of the $M$. avium pncA coding sequence. The 391 bp PCR fragment was labelled with $\left[{ }^{32} \mathrm{P}\right] \mathrm{dCTP}$ using the Random Primers DNA Labelling System (Gibco-BRL) according to the manufacturer's protocol. The blot was probed with the ${ }^{32} \mathrm{P}$-labelled PCR fragment as a probe, washed under low stringency, and subjected to autoradiography.

Transformation of mycobacteria. The $p n c A$ plasmid construct for transformation of BCG and PZA-R (ATCC 35828, a PZAresistant derivative of $M$. tuberculosis strain $H 37 \mathrm{Rv}$ ) was made as follows. The $1.6 \mathrm{~kb} \mathrm{BamHI}$ fragment containing the $M$. avium pncA gene was cloned into the KpnI site of the hygromycin mycobacterial shuttle vector p16R1 (Garbe et al., 1994) as described by Sambrook et al. (1989). The p16R1$1.6 \mathrm{~kb} \mathrm{M}$. avium pncA construct and the same vector harbouring the $M$. tuberculosis pncA gene on a $3.2 \mathrm{~kb}$ EcoRI-Pst I fragment, along with the vector control, were transformed by electroporation into the naturally PZAresistant $M$. bovis BCG and PZA-R using the method described previously (Zhang et al., 1993). The PZA-R isolate has been shown in a previous study to contain a single $G$ deletion at nucleotide position 288 of the $M$. tuberculosis pncA sequence (Scorpio \& Zhang, 1996).

Western blot analysis. This was performed as described previously (Zhang et al., 1991). The rabbit polyclonal antibody used was raised against the purified $M$. tuberculosis PZase protein overexpressed in E. coli (unpublished data), and this antibody was found to cross-react with the $M$. avium PZase protein.

Overexpression of $\boldsymbol{M}$. avium PZase protein in $\boldsymbol{E}$. coli. The $M$. avium PZase protein was overexpressed by a PCR approach using the following primers. The forward primer (5'GCTCGTTGTCGAGCAAACCG3') was taken from 93 bp upstream of the $M$. avium pncA gene start codon, and the reverse primer ( $5^{\prime}$ TGTCGGATCCAGGGGCGCCA3') was taken from 23 bp downstream of the stop codon of the $M$. avium pncA gene. The PCR was performed as described by Saiki $e t$ al. (1988) with the cloned $1.6 \mathrm{~kb}$ BamHI-pUC19 construct as a template using the following parameters: $95^{\circ} \mathrm{C}$ for $5 \mathrm{~min}$, followed by 30 cycles of $95^{\circ} \mathrm{C} 1 \mathrm{~min}, 55^{\circ} \mathrm{C} 1 \mathrm{~min}$ and $72^{\circ} \mathrm{C} 1$ min. The PCR product containing the $M$. avium pncA gene was cloned into pCR2.1 vector using the TA Cloning Kit (Invitrogen). The recombinant $E$. coli clone overexpressing the PZase enzyme was identified by restriction digestion of the plasmid DNA and by assaying for enhanced PZase enzyme activity conferred by the insert DNA using the PZase assay as described above. The $M$. tuberculosis pncA gene was similarly overexpressed and included as a control for comparison with the $M$ avium pnc $A$.

\section{RESULTS}

\section{PZA susceptibilities of various mycobacterial species}

Heifets et al. (1986) found that M. avium was naturally resistant to more than $100 \mu \mathrm{gZA} \mathrm{ml}^{-1}$, a concentration to which $M$. tuberculosis is susceptible; however, the exact MIC for $M$. avium was not determined. We therefore determined the MIC of $M$. avium along with those of some fast-growing mycobacteria (M. smegmatis, M. vaccae) and E. coli, to shed some light on the relationship between susceptibility to PZA and PZase activity. The MIC of PZA for $M$. avium was found to be about $500 \mu \mathrm{g} \mathrm{ml}^{-1}$, whereas $M$. smegmatis, M. vaccae and E. coli were all resistant to more than $2000 \mu \mathrm{g} \mathrm{PZA}$ $\mathrm{ml}^{-1}$. Thus the MIC for $M$. avium is intermediate between those for M. tuberculosis and fast-growing mycobacteria.

\section{Cloning of the $M$. avium pncA gene}

In a preliminary Southern blot analysis, genomic DNA from $M$. avium was found to hybridize with the $M$. tuberculosis pncA probe (data not shown). To clone the $M$. avium pncA gene, we screened an $M$. avium $\lambda$ ZAPII DNA library using the 329 bp PCR product containing part of the $M$. tuberculosis pncA gene as a probe. Because the $M$. avium phage library was constructed by ligating into the vector 4-8 kb genomic DNA fragments which were sonicated, blunt-ended and added with EcoRI linker, the insert DNA can be released with EcoRI. The DNA from the identified positive phage clone was digested with EcoRI and a $5.8 \mathrm{~kb} E c o \mathrm{RI}$ insert was obtained. Restriction mapping and Southern blotting analysis of the phage DNA localized the M. avium pncA gene on a $1.6 \mathrm{~kb}$ Bam HI subfragment within the $5.8 \mathrm{~kb}$ insert using the $M$. tuberculosis $p n c A$ probe. This $1.6 \mathrm{~kb} \mathrm{BamHI}$ fragment was subcloned into pUC19 for DNA sequence analysis.

\section{Sequence analysis of the $M$. avium pncA gene}

The $M$. avium $p n c A$ sequence was determined on both strands for the $1.6 \mathrm{~kb} \mathrm{BamHI}$ pUC19 construct using universal forward and reverse primers as well as primers derived from within the $1.6 \mathrm{~kb} B a m \mathrm{HI}$ fragment. The $M$. avium pncA gene, which is located at $248 \mathrm{bp}$ downstream from the $5^{\prime}$ end of the $1.6 \mathrm{~kb}$ fragment, was found to be $561 \mathrm{bp}$ long; this would encode a polypeptide of 187 amino acids with a predicted molecular mass of $19785.91 \mathrm{Da}$. The predicted $M$. avium PncA protein is only one amino acid longer than the corresponding M. tuberculosis enzyme (186 amino acids, GenBank accession number U59967). Sequence comparisons showed that the $M$. avium PncA has about $67.7 \%$ and $32.8 \%$ amino acid identity with the corresponding $M$. tuberculosis and $E$. coli enzymes, respectively (Fig. 1).

Translation of both upstream and downstream regions of the $p n c A$ did not reveal significant open reading frames with homology to known proteins in the databases. It is noteworthy that the upstream and downstream DNA sequence of the $M$. avium $p n c A$ did not show any significant homology with the corresponding regions in M. tuberculosis (data not shown). In $E$. coli, pncA is located immediately downstream of the L-asparginase I gene (asnA) and forms an operon with ansA (Jerlstrom et al., 1989). However, the 
$\begin{array}{lllllll}15 & 16 & 30 & 31 & 45 & 46 & 60\end{array}$

$1 \mathrm{M}$. avium PnCA M--RMIIVDVQSDI CECOSVPVAGGAAVA PPSTPTWTTLPGYDY VVVATQDFEIDPGDH

$2 M$. tb PncA

3 E. coli PncA

M--RAIIIVDVQRDF MPPRNILLVDLORTPF

CECOSLAVTGCAALA RAISDYLAEAADYHH -VVATKDFEIDPGDH CACGALAVPEODSTV DVANRLIDWCQSRGE AVIASQDWEPANHGS

1 M. avium PnCA

2 M. tb PncA

3 E. coli PncA

61 7576

9091

105106

120

IS-----DRP--- D--YSSSWPAECLAG SAGADFRPELDTTRV DAVRRG--AYAAGY

FS------GTP--- D--YSSSWPPECVSG TPGXDFHPSLDTSAI EAVYYRO--AYTGAY

FASQHGVEPYTPGQL DGLPQTFWPDECVQN SEGAQLHPLLHQKAI AAVPHKGENPLVDSY

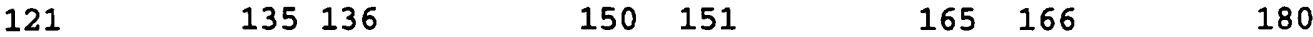

8GFEGVDD-NGTPLL EWLRRRGVDIVDVVG IATDHCVRRTAEDAA RAGLTTRVLVHLTAA

SGTEGVDE-NGTPLL NWLRQRGVDEVDVVO IATDHCVRQTAEDAV RNGLATRVLVDLTAG SAFFDNGRRQKTSLD DWLRDHEIDELIVMO LATDYCVKFTVLDAL QLOYKVNVITDGCRG

M. avium PnCA

2 M. tb PnCA

3 E. coli PnCA

$181 \quad 195196 \quad 210211$

1 M. avium PncA

2 M. tb PnCA

AAEDS--AARALDIA RSAGIEIVGAR---- ---

187

VSADT--TVARLEA RTASVEIVCSS---- ---

186

3 E. Coli PncA

VNIQPQDSAHAFMEM SAMGATLYTLADWEE TQG

213

Fig. 1. Comparison of the $M$. avium PZase (PncA) amino acid sequence with those of $M$. tuberculosis (M. tb) and $E$. coli. Gaps in the sequence alignment were introduced automatically by the CLUSTAL W method to maximize the sequence homology. Identical amino acid residues in all three sequences are in bold type.

upstream sequence of $M$. avium pncA did not have any significant homology to the $E$. coli ans $A$, indicating that the organization of the $M$. avium $p n c A$ and its upstream sequence is different from that in E. coli.

\section{PZase expression in $M$. avium}

To determine the level of PZase expression and investigate its possible regulation, we performed Western blot analysis with protein extracts of $M$. avium and $M$. tuberculosis grown under various conditions, i.e. acid $\mathrm{pH}(5.5)$ and with the enzyme substrates nicotinamide and PZA, using a rabbit polyclonal antibody raised against the $M$. tuberculosis PZase protein. Both $M$. avium and $M$. tuberculosis were found to constitutively express a very low amount of PZase protein, hardly detectable by Western blotting (data not shown). The level of PZase expression in M. avium was comparable to that in $M$. tuberculosis. Acid $\mathrm{pH}(5 \cdot 5)$ or growth in presence of $50 \mu \mathrm{g} \mathrm{ml}^{-1}$ of the PZase substrates nicotinamide and PZA did not significantly induce the production of PZase in $M$. avium or in M. tuberculosis as judged by Coomassie staining of SDS-PAGE gels and Western blot analysis (data not shown).

\section{Presence of pncA homologues in other mycobacteria}

To investigate whether there are $p n c A$ homologues in other mycobacteria, a Southern blot was performed with a portion of the $M$. avium pncA gene as a probe on BamHI-digested genomic DNA from a panel of mycobacterial species (Fig. 2). The $M$. avium pncA probe hybridized significantly with genomic DNA from $M$. terrae $(6.4 \mathrm{~kb}$, lane 4$), M$. gastri $(1.6 \mathrm{~kb}$, lane 5$), M$. marinum $(1.6 \mathrm{~kb}$, lane 6$), M$. fortuitum $(1.2 \mathrm{~kb}$, lane 7$)$, M. gordonae (1 kb, lane 10), M. szulgai $(6 \cdot 2 \mathrm{~kb}$, lane 11$)$,

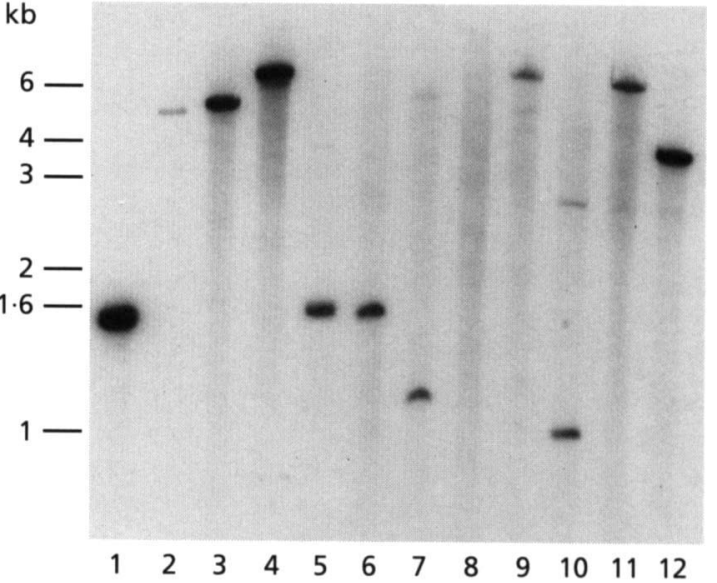

Fig. 2. Presence of pncA homologues in mycobacteria detected by Southern blotting. Genomic DNA from various mycobacterial species was digested with BamHI, transferred to nylon membrane, and the blot was probed with the ${ }^{32}$ p-labelled PCR fragment containing part of the $M$. avium pncA gene (see Methods). Lanes: 1, $M$. avium; $2, M$. bovis BCG; $3, M$. tuberculosis H37Rv; 4, M. terrae; 5, M. gastri; $6, M$. marinum; 7 , $M$. fortuitum; $8, M$. chelonae; $9, M$. xenopi; $10, M$. gordonae; 11 , M. szulgai; 12 , M. celatum.

M. celatum $(3.8 \mathrm{~kb}$, lane 12$)$ and M. kansasii $(1.8 \mathrm{~kb}$, not shown); it hybridized less intensely with $M$. xenopi $(6.4 \mathrm{~kb}$, lane 9$)$, and only poorly with DNA from $M$. chelonae. M. smegmatis did not give significant hybridization with the $M$. avium $p n c A$ probe (data not shown).

\section{M. avium pncA confers PZA susceptibility to PZA- resistant $M$. tuberculosis complex organisms}

Naturally PZA-resistant BCG and M. tuberculosis strains with acquired PZA-resistance lack or have 
Table 1. Susceptibilities of mycobacterial strains to PZA

\begin{tabular}{|c|c|c|c|c|}
\hline \multirow[t]{2}{*}{ Strain } & \multicolumn{4}{|c|}{ MIC of PZA ( $\mu \mathrm{g} \mathrm{ml}^{-1}$ ) } \\
\hline & No plasmid & pl6Rl & $\begin{array}{c}\text { M. avium } \\
\text { pncA }\end{array}$ & M. tb.pncA \\
\hline M. avium & 500 & ND & ND & ND \\
\hline BCG & $>500$ & $>500$ & $50-100$ & $50-100$ \\
\hline M. $t b$. PZA-R & $>500$ & $>500$ & $50-100$ & $50-100^{*}$ \\
\hline M. $t b . \mathrm{H} 37 \mathrm{Rv}$ & $50-100$ & ND & ND & ND \\
\hline
\end{tabular}

ND, Not done. M. tb., M. tuberculosis.

* Data from Scorpio \& Zhang (1996).

reduced PZase activity due to $p n c A$ mutations (Scorpio \& Zhang, 1996; Scorpio et al., 1997a, b; Sreevatsan et al., 1997). To determine if the $p n c A$ gene from M. avium would be functionally expressed and confer PZA sensitivity, we transformed BCG and PZA-R, a PZAresistant $M$. tuberculosis isolate (PZA-R) derived from strain $\mathrm{H} 37 \mathrm{Rv}$, with a hygromycin plasmid construct harbouring the $1.6 \mathrm{~kb} \mathrm{BamHI}$ fragment containing the $M$. avium pncA gene. The $M$. avium $p n c A$ gene in the $1.6 \mathrm{~kb} \mathrm{BamHI}$ fragment has $248 \mathrm{bp}$ upstream sequence from the start codon, which should contain the promoter for the M. avium pncA. The M. tuberculosis pncA gene on a $3.2 \mathrm{~kb}$ EcoRI-PstI fragment (Scorpio \& Zhang, 1996) which was cloned into the same hygromycin vector was used as a positive control, and the vector alone as a negative control. Interestingly, the $M$. avium $p n c A$ construct gave functional expression of PZase activity in both BCG and PZA-R, and conferred PZA susceptibility in both organisms. Both BCG and PZA-R are resistant to more than $500 \mu \mathrm{g} \mathrm{PZA} \mathrm{ml}{ }^{-1}$, and transformation with the $M$. avium $p n c A$ gene made them susceptible to $50-100 \mu \mathrm{g} \mathrm{PZA} \mathrm{ml}{ }^{-1}$ (Table 1). The above data indicate that the promoter of the $M$. avium $p n c A$ gene is recognized by the M. tuberculosis and $M$. bovis transcriptional and translational apparatus and that the $M$. avium PZase has the ability to potentiate PZA action. The level of PZA susceptibility conferred by the $M$. avium $p n c A$ gene was comparable to that conferred by the M. tuberculosis pncA construct (MIC 50-100 $\mu \mathrm{g} \mathrm{PZA} \mathrm{ml}{ }^{-1}$ ). Western blot analysis of the recombinant BCG strains revealed that the $M$. $t u$ berculosis pncA gave a protein band of about $19 \cdot 8 \mathrm{kDa}$ as expected; however, the $M$. avium $p n c A$ produced a protein band with a size of about $24 \mathrm{kDa}$ (Fig. 3), bigger than the $19 \cdot 8 \mathrm{kDa}$ predicted from the DNA sequence.

\section{Investigation of anomalous mobility of the $M$. avium PZase protein on Western blots}

Because the $M$. avium PZase migrated as a $24 \mathrm{kDa}$ band on Western blots, as opposed to a $19 \cdot 8 \mathrm{kDa}$ band as expected, we wondered if this was due to glycosylation of the $M$. avium PZase. However, incubation of the protein extracts containing $M$. avium $\mathrm{PZase}$ with the enzyme Endo H (Sigma), which removes carbohydrates

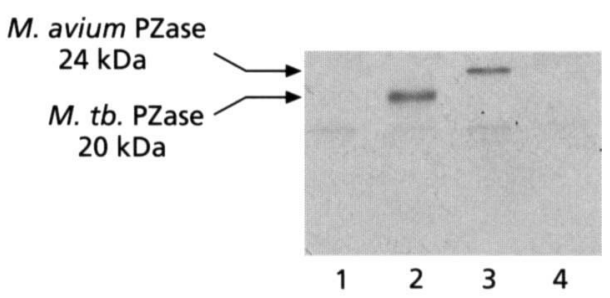

Fig. 3. Western blot analysis, using a rabbit polyclonal antibody raised against the $M$. tuberculosis PZase protein, of the expression of the $M$. avium PZase in BCG. Lanes: 1, BCG transformed with vector control p16R1; 2, BCG transformed with the $M$. tuberculosis pncA gene; 3 , BCG transformed with $M$. avium pncA gene; 4, BCG transformed with vector control p16R1.

from glycoproteins, did not alter the mobility of this protein on Western blots (data not shown), indicating that the slower migration of M. avium PZase on SDSPAGE gels is not due to glycosylation of this protein.

To obtain more direct evidence that the slower migration of $M$. avium PZase on SDS-PAGE gels is not due to glycosylation, we overexpressed the $M$. avium $p n c A$ gene as well as the $M$. tuberculosis $p n c A$ gene as a control in $E$. coli, a host that is not known to glycosylate proteins. Both the overexpressed $M$. avium and $M$. tuberculosis PZase proteins gave functional enzyme activity in E. coli (data not shown). The same migration pattern as in Fig. 3 was observed, i.e. the overexpressed $M$. avium PZase still migrated more slowly than the corresponding overexpressed $M$. tuberculosis enzyme included as a control on a Western blot (data not shown). This strongly suggests that the retarded mobility of the M. avium PZase is not due to glycosylation.

\section{DISCUSSION}

In this study, we have cloned and characterized the $M$. avium pncA gene encoding PZase. We have demonstrated that $M$. avium, which is naturally resistant to PZA, has a functional $p n c A$ gene that confers PZA susceptibility to PZA-resistant $M$. tuberculosis complex organisms. Sequence analysis showed that the $M$. avium 
PZase has a high degree of homology $(67.7 \%$ amino acid identity) with the $M$. tuberculosis enzyme, a finding consistent with the strong hybridization of $M$. avium $p n c A$ with the corresponding $M$. tuberculosis gene. Southern blot analysis indicated that there are close pncA homologues in the following mycobacterial species: M. terrae, M. gastri, M. marinum, M. fortuitum, M. gordonae, $M$. celatum, M. xenopi, M. szulgai and $M$. kansasii. However, $M$. chelonae and $M$. smegmatis did not produce significant hybridization with the $M$. avium pncA gene; the lack of significant hybridization in these organisms is probably due to poor homology of their $p n c A$ genes with the $M$. avium $p n c A$, rather than to the absence of a pncA gene, since these mycobacteria have been shown to have functional PZase activity (Tarnok \& Rohrscheidt, 1976; Koneman et al., 1988). It is interesting to note that $M$. celatum gave strong hybridization signals with the $M$. avium pncA gene, a finding consistent with the similarity of these two organisms in terms of $16 \mathrm{~S}$ rRNA sequence homology and biochemical properties used to identify the species (Butler et al., 1993). An unexpected finding is that $M$. xenopi, which is taxonomically close to $M$. avium and M. celatum (Butler et al., 1993), gave a relatively weak hybridization signal with the M. avium $p n c A$ gene. On the other hand, $M$. fortuitum, a fast-growing mycobacterium, produced a relatively strong hybridization signal. It is worth noting that unlike many other mycobacteria, $M$. kansasii has an amidase with only nicotinamidase activity but no PZase activity (Tarnok \& Rohrscheidt, 1976), yet it produced a $1.8 \mathrm{~kb}$ Bam HI fragment which hybridized with the $M$. avium $p n c A$ probe by Southern blot analysis. Work is under way to characterize the $M$. kansasii nicotinamidase gene and to determine the genetic basis for the dissociation of PZase and nicotinamidase activities in the M. kansasii enzyme.

A somewhat unexpected finding is that $M$. avium PZase when overexpressed in BCG gave a higher molecular mass band $(24 \mathrm{kDa})$ than that predicted from its DNA sequence $(19.8 \mathrm{kDa})$ on Western blots; by contrast, the M. tuberculosis PZase, which is only one amino acid shorter than the M. avium PZase, gave a $19 \cdot 8 \mathrm{kDa}$ band as expected based on its DNA coding sequence. The retarded mobility of the $M$. avium PZase cannot be due to glycosylation for two reasons: (1) overexpression of the $M$. avium PZase in E. coli, which is not known to glycosylate proteins, still gave the same mobility pattern as in BCG; and (2) digestion of the M. avium PZase with Endo $\mathrm{H}$, which removes carbohydrates from glycoproteins, did not change the mobility of the protein on Western blots. The possibility that the M. avium PZase used an alternative upstream start codon and encoded a larger protein can also be excluded, because there is a stop codon immediately upstream of the start codon of the $M$. avium $p n c A$ gene. Very basic proteins such as histones with considerably more positively charged amino acids migrate more slowly on SDS-PAGE gels than expected from their molecular mass (Panyim \& Chalkley, 1971). The M. avium PZase has more overall positively charged amino acid residues than the $M$. tuberculosis enzyme: 21 positively charged (with 32 negatively charged) versus 17 positively charged (with 34 negatively charged). The more positive overall charge of the M. avium PZase is likely to be the cause of its slower migration on the Western blots.

In the present study, we have shown that the M. avium $p n c A$ gene conferred PZA susceptibility in M. tuberculosis complex organisms. This is surprising because $M$. avium itself is not susceptible to PZA (MIC $500 \mu \mathrm{g}$ PZA $\mathrm{ml}^{-1}$ versus $50 \mu \mathrm{g} \mathrm{PZA} \mathrm{ml}^{-1}$ in $M$. tuberculosis); and the fact that the $M$. avium pncA conferred PZA susceptibility to resistant $M$. tuberculosis strains suggests that the relative nonsusceptibility to PZA of the $M$. avium species is not because it has an ineffective PZase enzyme. The low-level expression of the $M$. avium PZase could not have contributed to its natural resistance to PZA, as its level of expression is comparable to that in susceptible $M$. tuberculosis (data not shown). There are three possible reasons, which are not necessarily mutually exclusive, for the nonsusceptibility of $M$. avium to PZA. The first possibility is that $M$. avium has a more active efflux mechanism that pumps out POA than $M$. $t u$ berculosis. $M$. smegmatis has been shown to have an efflux pump LfrA (Takiff et al., 1996), and it is not clear whether $M$. avium would also have a similar efflux mechanism involved in pumping out certain antibiotics including POA. Further studies will be necessary to determine whether the natural resistance of $M$. avium to PZA is due to a more active efflux mechanism for pumping out POA. The second possibility is that $M$. avium, unlike $M$. tuberculosis, may not have a sensitive target for POA or have a target that is less susceptible to POA than that in M. tuberculosis; however, because the target for POA in M. tuberculosis has not yet been identified, this possibility cannot be addressed at this time.

The third possibility is that the cell wall of $M$. avium may not take up PZA or its derivative POA as efficiently as that of $M$. tuberculosis. Indeed, we have some data indicating that the $M$. avium cell wall does not take up as much $\left[{ }^{14} \mathrm{C}\right] \mathrm{POA}$ as that of $M$. tuberculosis under acid $\mathrm{pH}$ conditions (unpublished). This difference in the cell wall permeability to POA is consistent with the finding that $M$. avium is less susceptible than $M$. tuberculosis to certain antibiotics such as POA esters (Cynamon et al., 1995; Yamamoto et al., 1995) and aminoglycosides (Jarvier \& Nikaido, 1994). Another example showing that significant differences exist among the cell walls of different mycobacteria in terms of their permeability to certain antibiotics is that the cell wall of $M$. chelonae is much less permeable to the $\beta$-lactam antibiotic cephalosporins than that of $M$. tuberculosis (Jarvier \& Nikaido, 1994). Our data on the differential permeability of mycobacterial cell walls to POA are consistent with these findings. It will be interesting to analyse further the relationship between cell wall permeability and susceptibility to PZA/POA using reagents that alter the cell wall permeability, and to identify the structural basis of the cell wall differences between $M$. avium and $M$. tuberculosis that may be involved in the uptake of POA. 


\section{ACKNOWLEDGEMENTS}

This work was supported by a Faculty Development Award (to Y.Z.) from The Johns Hopkins School of Hygiene and Public Health, and in part by a grant from the American Lung Association. We thank Salman Siddiqi for provision of mycobacterial strains, and acknowledge the receipt of $\lambda$ ZAPII $M$. avium phage DNA library from NIH AIDS Reagent Program, Division of AIDS, NIAID, NIH. We also thank the reviewers of the paper for their constructive comments.

\section{REFERENCES}

Bonicke, R. \& Lisboa, B. P. (1959). Typendifferenzierung der Tuberkulosebakterien mit Hilfe des Nikotinamidasetests. Tuberkuloseartzt 13, 375-377.

Butler, W. R., O'Connor, S. P., Yakrus, M. A. \& 8 other authors (1993). Mycobacterium celatum sp. nov. Int J Syst Bacteriol 43, 539-548.

Cynamon, M. H., Gimi, R., Gyenes, F. \& 7 other authors (1995). Pyrazinoic acid esters with broad spectrum in vitro antimycobacterial activity. J Med Chem 38, 3902-3907.

Elliner, J. J., Goldberger, M. J. \& Parenti, D. M. (1991). Mycobacterium avium infection and AIDS: a therapeutic dilemma in rapid evolution. J Infect Dis 163, 1326-1335.

Garbe, T., Barathi, J., Barnini, S., Zhang, Y., Abou-Zeid, C., Tang, D., Mukherjee, R. \& Young, D. B. (1994). Transformation of mycobacterial species using hygromycin resistance as selectable marker. Microbiology 140, 133-138.

Heifets, L. B., Iseman, M. D., Crowle, A. J. \& Lindholm-Levy, P. J. (1986). Pyrazinamide is not active against Mycobacterium avium complex. Am Rev Respir Dis 134, 1287-1288.

Jarlier, V. \& Nikaido, H. (1994). Mycobacterial cell wall: structure and role in natural resistance to antibiotics. FEMS Microbiol Lett 123, 11-18.

Jerlstrom, P. G., Bezjak, D. A., Jennings, M. P. \& Beacham, I. R. (1989). Structure and expression in Escherichia coli K-12 of the Lasparaginase I-encoding ans $A$ gene and its flanking regions. Gene $78,37-46$.

Koneman, E. W., Allen, S. D., Dowell, V. R., Janda, W. M., Sommers, H. M. \& Winn, W. C. (1988). Mycobacteria. In Color Atlas and Textbook of Diagnostic Microbiology, 3rd edn, pp. 535-572. Philadelphia: J. B. Lippencott Co.

Konno, K., Feldman, F. M. \& McDermott, W. (1967). Pyrazinamide susceptibility and amidase activity of tubercle bacilli. Am Rev Respir Dis 95, 461-469.

McClatchy, J. K., Tsang, A. Y. \& Cernich, M. S. (1981). Use of pyrazinamidase activity in Mycobacterium tuberculosis as a rapid method for determination of pyrazinamide susceptibility. Antimicrob Agents Chemother 20, 556-557.

McDermott, W. \& Tompsett, R. (1954). Activation of pyrazinamide and nicotinamide in acidic environment in vitro. Am Rev Tuberc 70, 748-754.

Miller, M., Thibert, L., Desjardins, F., Siddiqi, S. \& Dascal, A. (1995). Testing of susceptibility of Mycobacterium tuberculosis to pyrazinamide : comparison of Bactec method with pyrazinamidase assay. J Clin Microbiol 33, 2468-2470.
Panyim, S. \& Chalkley, R. (1971). The molecular weights of vertebrate histones exploiting a modified sodium dodecyl sulfate electrophoretic method. J Biol Chem 246, 7557-7660.

Saiki, R. K., Gelfand, D. H., Stoffel, S., Scharf, S. J., Higuchi, R., Horn, G. T., Mullis, K. B. \& Erlich, H. A. (1988). Primer-directed enzymatic amplification of DNA with a thermostable DNA polymerase. Science 239, 487-491.

Sambrook, J., Fritsch, E. F. \& Maniatis, T. (1989). Molecular Cloning: a Laboratory Manual, 2nd edn. Cold Spring Harbor, NY: Cold Spring Harbor Laboratory.

Scorpio, A. \& Zhang, Y. (1996). Mutations in $p n c A$, a gene encoding pyrazinamidase/nicotinamidase, cause resistance to the antituberculous drug pyrazinamide in tubercle bacillus. Nat Med 2, 662-667.

Scorpio, A., Collins, D. M., Whipple, D., Cave, D., Bates, J. \& Zhang, Y. (1997a). Rapid differentiation of bovine and human tubercle bacilli based on a characteristic mutation in the bovine pyrazinamidase gene. J Clin Microbiol 32, 106-110.

Scorpio, A., Lindholm-Levy, P., Heifets, L., Gilman, R., Siddiqi, S., Cynamon, M. \& Zhang, Y. (1997b). Characterization of $p n c A$ mutations in pyrazinamide-resistant Mycobacterium tuberculosis. Antimicrob Agents Chemother 41, 540-543.

Sreevatsan, S., Pan, X., Zhang, Y., Kreiswirth, B. \& Musser, J. M. (1997). Mutations associated with pyrazinamide resistance in pncA of Mycobacterium tuberculosis complex organisms. Antimicrob Agents Chemother 41, 636-640.

Takiff, H. E., Cimino, M., Musso, M. C., Weisbrod, T., Martinez, R., Delgado, M. B., Salazar, L., Bloom, B. R. \& Jacobs, W. R., Jr (1996). Efflux pump of the proton antiporter family confers low-level fluoroquinolone resistance in Mycobacterium smegmatis. Proc Natl Acad Sci USA 93, 362-366.

Tarnok, I. \& Rohrscheidt, E. (1976). Biochemical background of some enzymatic tests used for the differentiation of mycobacteria. Tubercle 57, 145-150.

Trivedi, S. S. \& Desai, S. G. (1987). Pyrazinamidase activity of Mycobacterium tuberculosis - a test of sensitivity to pyrazinamide. Tubercle 68, 221-224.

Wayne, L. G. (1974). Simple pyrazinamidase and urease tests for routine identification of mycobacteria. Am Rev Respir Dis 109, 147-151.

Yamamoto, S., Toida, I., Watanabe, N. \& Ura, T. (1995). In vitro antimycobacterial activities of pyrazinamide analogs. Antimicrob Agents Chemother 39, 2088-2091.

Zhang, Y., Lathigra, R., Garbe, T., Catty, D. \& Young, D. (1991). Genetic analysis of superoxide dismutase, the 23 kilodalton antigen of Mycobacterium tuberculosis. Mol Microbiol 5, 381391.

Zhang, Y., Garcia, M. J., Lathigra, R., Allen, B., Moreno, C., van Embden, J. D. A. \& Young, D. (1992). Alterations in the superoxide dismutase gene of an isoniazid-resistant strain of Mycobacterium tuberculosis. Infect Immun 60, 2160-2165.

Zhang, Y., Garbe, T. \& Young, D. (1993). Transformation with kat $G$ restores isoniazid sensitivity in Mycobacterium tuberculosis isolates resistant to a range of drug concentrations. Mol Microbiol $8,521-524$.

Received 19 March 1997; revised 9 June 1997; accepted 30 June 1997. 